\title{
Selección y uso de la información como competencia genérica en la formación profesional de Ingenieros de Sistemas
}

\author{
Luis Anderson Coronel-Rojas ${ }^{\mathrm{a} *}$ \\ ${ }^{a}$ Magister en Prácticas pedagógicas., Universidad Francisco de Paula Santander \\ Seccional, Ocaña- Colombia \\ Forma de citar: Coronel, L. A (2017). Selección y uso de la información como competencia genérica en \\ la formación profesional de Ingenieros de Sistemas. Perspectivas, 2(1). 6-17
}

Recibido el 3 de febrero de 2017

Aceptado el 16 de mayo de 2017

Disponible en Internet el 16 de junio de 2017

\begin{abstract}
Palabras clave
Competencia genérica, educación superior, selección y uso de la información, tecnologías de información.
\end{abstract}

Autor para correspondencia
lacoronelr@ufpso.edu.co

https://doi.org/10.22463/25909215.1280
Resumen: Las competencias genéricas han encontrado gran auge en el campo educativo actual. De hecho, un gran número de instituciones de educación superior las contemplan estructuralmente en sus planes de formación y las justifican en extensos documentos académicos. Sin embargo, hasta la fecha no existen suficientes reportes de investigación que reporten que estas competencias son efectivamente apropiadas en las instituciones de educación superior. Los estudiantes universitarios que egresan de sus carreras no parecen estar suficientemente preparados para los retos que se plantean en la vida real, carecen de muchas de las habilidades que les permitan tomar riesgos, organizar, e ir más allá de lo que han aprendido en clase, haciendo trasferencias de contenidos a situaciones nuevas, sobre todo cuando se trata de hacer una selección apropiada de la información que les sirva para la toma de decisiones. En esta dirección, este artículo muestra los resultados de un estudio realizado con estudiantes de primero y último semestre del programa de Ingeniería de Sistemas de la Universidad Francisco de Paula Santander Ocaña, en el que se relaciona la evaluación de un conjunto de habilidades relacionadas con la competencia que rige la selección y el uso de la información. Específicamente se busca indagar en la forma en la que los estudiantes de Ingeniería de Sistemas adoptan esta competencia a partir de las percepciones de docentes y otros estudiantes. Los resultados incluyen el análisis de subcategorías inductivas como la recopilación, evaluación y clasificación de la información relevante, el uso de los 


\section{Keywords \\ Generic competition, higher education, use and selection of information, information technologies.}

medios adecuados para transmitir y las habilidades en el uso de la tecnología de la información. En general los estudiantes destacan que esta competencia es importante para su formación, reconociendo que tienen la capacidad para seleccionar y clasificar información, que son creativos en el uso y selección del medio para transmitirla y que poseen habilidades de las se han podido apropiar durante su formación en el programa. Aun así, reconocen que todavía hay aspectos por mejorar.

\section{Use and selection of information as generic competition in the professional training of Systems Engineers}

Abstract :Generic competitions have become increasingly popular in the current educational field. In fact, they are considered by numerous higher education institutions when structuring their educational programs, as well as advocated in extensive academic work. However, thus far, not enough research reports demonstrating these competitions as indeed suited to higher education exist. University graduates do not seem sufficiently prepared for real-life challenges: they lack the skills needed to organize, take risks, and go beyond what was taught in class, tailoring their knowledge to new situations, especially in cases of correctly choosing the information necessary in order to make an informed decision. Accordingly, this article presents the results of a study published by students in their first and last semester of Systems Engineering at the University of Francisco de Paula Santander, Ocaña. The study examines the link between the ability to successfully select and use information with the evaluation of a certain skillset. Specifically, it seeks to investigate the way the students enter such a competition based on the perceptions of their teachers and peers. The results include the analysis of inductive subcategories, such as the gathering, classification and evaluation of relevant information and their ability to relay it through the appropriate channels, as well as their proficiency in information technology. Overall, the students assert that this competition is important for their education, and recognize their efficiency in selecting and classifying information, as well as their creativity the means by which they convey it, and the skills they have been able to successfully adapt during their education program However, they also recognize there are still points that require improvement- 


\section{Palavras chave}

Competência genérica, ensino superior, seleção e uso de informações, tecnologias da informação.

\section{Seleção e uso de informações como uma competência genérica na formação profissional de Engenheiros de Sistemas}

Resumo: As competências genéricas encontraram grande auge no atual campo educacional. $\mathrm{Na}$ verdade, um grande número de instituições de ensino superior as considera estruturalmente em seus planos de treinamento e justifica-os em extensos documentos acadêmicos. No entanto, até à data, não há relatórios de pesquisa suficientes que relatam que essas competências são efetivamente apropriadas em instituições de ensino superior. Os estudantes universitários que se formam em suas carreiras não parecem suficientemente preparados para os desafios que surgem na vida real, carecem de muitas habilidades que thes permitem assumir riscos, organizar e ir além do que aprenderam na aula, Transferências de conteúdo para novas situações, especialmente quando se trata de fazer uma seleção apropriada de informações que os ajude a tomar decisões. Nessa direção, este artigo mostra os resultados de um estudo realizado com alunos do primeiro e último semestre do programa de Engenharia de Sistemas da Universidade Francisco de Paula Santander Ocaña, na qual a avaliação de um conjunto de habilidades relacionadas ao concorrência que rege a seleção e uso de informações. Especificamente, procura investigar a forma como os alunos de Engenharia de Sistemas adotam essa competência com base nas percepções de professores e outros alunos. Os resultados incluem a análise de subcategorias indutivas, como a coleta, avaliação e classificação de informações relevantes, o uso de meios apropriados para transmitir e as habilidades na utilização da tecnologia da informação. Em geral, os alunos enfatizam que esta competência é importante para a sua formação, reconhecendo que eles têm a capacidade de selecionar e classificar a informação, que são criativos no uso e seleção do meio para transmiti-lo, e que possuem habilidades que eles conseguiram apropriar durante seu treinamento. no programa. Mesmo assim, eles reconhecem que ainda há aspectos a serem melhorados.

\section{Introducción}

Las competencias genéricas han cobrado gran fuerza en los últimos años, y lo demuestran la cantidad de estudios e investigaciones que alrededor del tema se han tejido alrededor del mundo entero. Una de las razones que han potenciado este interés radica en la preocupación acerca de si se están formando profesionales capacitados y pertinentes para las sociedades actuales y si los modelos curriculares contemplan estrategias para el desarrollo de estas competencias.

A finales de los noventa empezaron a emerger numerosos informes sobre la educación superior y la realidad frente a los resultados de cómo los profesionales egresados de las diferentes carreras estaban enfrentando sus responsabilidades en sus distintos ambientas laborales. Como ejemplos, se pueden señalar investigaciones como el Proyecto Tuning (Comisión Europea, 2006), el mismo 
proyecto Tuning replicado para América Latina (comisión Europea, 2007) o el Informe Delors (1995), entre otros.

El proyecto Tuning, (2006) surge en Europa como una preocupación por el tema de las competencias en la educación superior. En él participaron 29 países del continente. En el estudio elaborado se establecieron 27 competencias, pero el estudio se concentró básicamente en la descripción de las competencias específicas, relacionadas directamente con la parte disciplinar, y las genéricas, que son aquellas transversales a cualquier profesional; entre ellas se pueden mencionar la capacidad o habilidad en la planeación, la creatividad y la habilidad para buscar, procesar y analizar información. Esta última constituye el foco de interés en la presente investigación.

El proyecto Tuningse desarrolla en América Latina, según afirman González, WagenaaryBeneitone (2004), como una réplica del desarrollado en Europa, con la finalidad de conocer el estado de las competencias a través del análisis realizado desde diferentes universidades de América. En el proyecto participaron 19 países, entre los cuales se encuentran Brasil, México, Chile, Argentina, y Colombia. El interés de los gobiernos de estas naciones estuvo en fortalecer el desarrollo de titulaciones y facilitar la articulación entre los distintos sistemas de educación superior.

En Colombia, el Ministerio de Educación Nacional (MEN, 2002) define cinco objetivos iniciales, con la finalidad de mejorar la articulación entre los distintosniveles de la educación formal (inicial, básica, media y superior) y de poder establecer referentes comunes para garantizar una educación de calidad en el país. Estos objetivos están direccionados a orientar la incorporación en todos los planes de estudio de los conocimientos, habilidades y valores requeridos para el desempeño ciudadano y productivo en igualdad de condiciones; garantizar el acceso de todos los estudiantes a estos aprendizajes; mantener los elementos esenciales de unidad nacional en el marco de una creciente descentralización; la comparabilidad con lo que los estudiantes aprenden en otros países y facilitar la transferencia de estudiantes entre centros educativos, regiones, y países.

La investigación que reporta este artículo se circunscribe al estudio de competencias en la educación superior colombiana, más concretamente, a la competencia genérica de selección y uso de la información en el Programa de Ingeniería de Sistemas de la Universidad Francisco de Paula Santander con sede en Ocaña. Esta Institución (UFPS, 2015) tiene como misión "Formar profesionales integrales en Ingeniería, ciencias de la computación, ingeniería de software e infraestructura de tecnología de la información (TI) con una sólida formación humanística que responda a los problemas actuales y futuras necesidades de la región, para enfrentar los retos tecnológicos acordes con la nueva sociedad de la información y del conocimiento".

Para que estos Ingenieros de Sistemas estén preparados para asumir los retosde la nueva sociedad del conocimiento que expresan en su misión, deben poseer habilidades para transmitir la información, seleccionar el medio idóneo para hacerlo, poseer habilidades en el uso de las TIC, capacidad para seleccionar y clasificar información, todo lo cual constituyen facetas de la competencia selección y uso de la información, concebida como núcleo de interés en la presente investigación.

Dado que las competencias genéricas, han pasado a ser un tema de vital importancia en la educación superior, el presente estudio busca indagar en la forma cómo los estudiantes de Ingeniería de Sistemas adoptan y desarrollan la competencia de selección y uso de la información. Concretamente, se busca analizar las percepciones de docentes y estudiantes sobre las habilidades alcanzadas en relación a esta competencia.

\section{Materiales y métodos}

La investigación fue de tipo descriptivoevaluativo, teniendo en cuenta el sistema curricular propio de la carrera. Por lo tanto, se 
emplearon elementos cualitativos y cuantitativos de la investigación, pero dado que sobresalen los argumentos, discusiones y conclusiones, predominó el diseño cualitativo. Asimismo, se consideró el modelo constructivista social, que el que institucionalmente ha sido adoptado por la UFPS Ocaña para sus procesos pedagógicos.

\subsection{Población y muestra}

La población objeto de estudio fueron los estudiantes del programa Ingeniería de Sistemas, de la Universidad Francisco de Paula Santander Ocaña. Para la muestra fueron seleccionados algunos estudiantes, tomados de los listados oficiales de la universidad a los que se tuvo acceso. Se escogieron 20 estudiantes de primer semestre y 20 estudiantes de décimo semestre, que es el último, del programa de Ingeniería de Sistemas.

\subsection{Fases del proyecto}

La recolección de datos de la investigación estuvo diseñada en tres fases:

\section{Primera fase: Cuestionario.}

Como una herramienta cuantitativa se elaboró un cuestionario cuyo propósito consistió en recoger información acerca de la percepción y opinión de los estudiantes en relación con los distintos elementos asociados a la competenciade selección y uso de la información. El instrumento se confeccionó sobre el modelo de escala Likert.

Para la aplicación del cuestionario se solicitó a algunos docentes del programa que permitieran la actividad durante su clase. Previamente, se dio a docentes y alumnos la información e instrucciones relacionadas con el objetivo de la investigación. El tiempo de administración del instrumento fue de 30 minutos.

\section{Segunda fase: Pintura enriquecida.}

Se solicitó a los estudiantes tanto de primer como de último semestre organizarse en grupos para esta actividad, que consistía en darles una pregunta orientadora para que fuera desarrollada mediante el diseño de una cartelera con la utilización de diferentes materiales suministrados por los investigadores y posteriormente realizar por parte de los mismos investigadores una serie de preguntas para corroborar los resultados plasmados en dicha cartelera, asociadas no solamente a la selección y uso de la información sino a las competencias, contempladas estas como atributos, habilidades y cualidades que debe tener un ingeniero de sistemas para el ejercicio de su profesión.

Como es natural, se marcaron como una estrategia de organización algunas reglas asociadas a los tiempos, la metodología y el producto esperado por los investigadores de los diferentes grupos. De acuerdo con lo que fueron argumentando los estudiantes, los investigadores formularon una serie de preguntas con la intención de ahondar en la recolección de información y tratando de generar una discusión alrededor del tema.

\section{Tercera Fase. Entrevista a los docentes}

Se llevó a cabo la entrevista semiestructurada a una muestra de docentes de primer y último semestre del programa de Ingeniería de Sistemas mediante un cuestionario que contiene de manera organizada y estructurada una serie de preguntas sobre las categorías inductivas seleccionadas y definidas por los investigadores, lo cual permitió conocer las opiniones y percepciones sobre los diferentes aspectos tratados de la investigación.

El tiempo previsto para el desarrollo de este instrumento fue de una hora. S los docentes se solicitó autorización para grabar las entrevistas. Como preámbulo se les explicó el objetivo de dichas entrevistas y como sería la dinámica del ejercicio. 
La gran mayoría se mostró atento y dispuesto a colaborar; otros no tanto. Para el caso de quienes no se mostraron reacios a participar, se recurrió a otros docentes con mayor voluntad para lograr mayor efectividad en los resultados.

\section{Resultados y discusión}

La figura 1 muestra las características demográficas de los estudiantes, según el semestre que cursan y los rangos de edad.

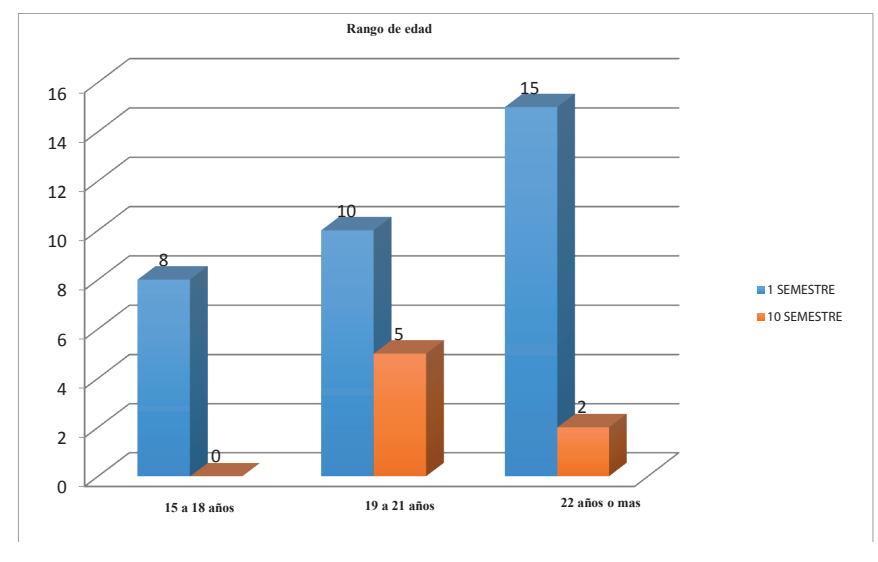

Figura 1

Análisis demográfico según rango de edad Fuente: Proceso de investigación
De acuerdo con la figura 1, se evidencia que un importante número de alumnos, que alcanza el $40 \%$ de los alumnos de primer semestre, se encuentran entre los 15 y 18 años, o sea, que son todavía menores de edad, principalmente porque están recién graduados de la básica secundaria. Un $50 \%$ está entre los 19 a 21 años; esto se debe a que son estudiantes que han tenido dificultades para ingresar a la carrera, o han decido trasladarse de otras carreras en las cuales ingresaron por segunda opción. El 10\% restante de los estudiantes tiene 22 años o más.

En el grupo de alumnos de décimo semestre, el rango de edad que prevalece es aquel donde están los estudiantes mayores de 22 años, lo cual significa que demográficamente constituyen un grupo análogo al de primer semestre después de los cinco años de carrera.

Con respecto al cuestionario aplicado a los estudiantes, la tabla 1 recoge los ítems que se les propusieron en relación con su percepción y opinión sobre la selección y uso de la información.

Tabla 1

Ítems del cuestionario

\begin{tabular}{|c|c|c|c|c|c|c|c|}
\hline Ítem \# & Descripción & Sem & $\begin{array}{l}\text { Totalmente } \\
\text { en } \\
\text { desacuerdo/ } \\
\text { nunca }\end{array}$ & $\begin{array}{l}\text { En } \\
\text { desacuerdo/casi } \\
\text { Nunca }\end{array}$ & $\begin{array}{l}\text { Indiferente/ } \\
\text { Algunas veces }\end{array}$ & $\begin{array}{l}\text { Desacuerdo/ } \\
\text { Casi Siempre }\end{array}$ & $\begin{array}{l}\text { Totalmente } \\
\text { desacuerdo/ } \\
\text { siempre }\end{array}$ \\
\hline \multirow[t]{2}{*}{1} & \multirow{2}{*}{$\begin{array}{l}\text { Evalúo la } \\
\text { información que } \\
\text { es apropiada, } \\
\text { para la toma de } \\
\text { decisiones }\end{array}$} & $1^{\circ}$ & 0 & $5 \%$ & $30 \%$ & $45 \%$ & $20 \%$ \\
\hline & & $10^{\circ}$ & $10 \%$ & $20 \%$ & $25 \%$ & $45 \%$ & $0 \%$ \\
\hline \multirow{2}{*}{2} & \multirow{2}{*}{$\begin{array}{l}\text { Archivo la } \\
\text { información de } \\
\text { la manera mas } \\
\text { adecuada. }\end{array}$} & $1^{\circ}$ & 0 & $10 \%$ & $15 \%$ & $55 \%$ & $20 \%$ \\
\hline & & $10^{\circ}$ & 0 & $20 \%$ & $30 \%$ & $40 \%$ & $10 \%$ \\
\hline \multirow{2}{*}{3} & \multirow{2}{*}{$\begin{array}{l}\text { Clasifico la } \\
\text { información } \\
\text { disponible para } \\
\text { facilitar el } \\
\text { acceso cuando se } \\
\text { necesite } \\
\text { recuperarla }\end{array}$} & $1^{\circ}$ & 0 & $5 \%$ & $20 \%$ & $64 \%$ & $10 \%$ \\
\hline & & $10^{\circ}$ & 0 & $10 \%$ & $30 \%$ & $40 \%$ & $20 \%$ \\
\hline
\end{tabular}




\begin{tabular}{|c|c|c|c|c|c|c|c|}
\hline \multirow{2}{*}{4} & \multirow{2}{*}{$\begin{array}{l}\text { Para transmitir la } \\
\text { información } \\
\text { generalmente se } \\
\text { hace uso del } \\
\text { medio apropiado }\end{array}$} & $1^{\circ}$ & 0 & $5 \%$ & $40 \%$ & $50 \%$ & $5 \%$ \\
\hline & & $10^{\circ}$ & $5 \%$ & 0 & $45 \%$ & $40 \%$ & $10 \%$ \\
\hline \multirow{2}{*}{5} & \multirow{2}{*}{$\begin{array}{l}\text { Para tratar una } \\
\text { situación es } \\
\text { necesario } \\
\text { obtener } \\
\text { información } \\
\text { relevante } \\
\end{array}$} & $1^{\circ}$ & 0 & 0 & $25 \%$ & $50 \%$ & $25 \%$ \\
\hline & & $10^{\circ}$ & 0 & 0 & $10 \%$ & $55 \%$ & $35 \%$ \\
\hline \multirow[t]{2}{*}{6} & \multirow{2}{*}{$\begin{array}{l}\text { Poseo } \\
\text { habilidades en el } \\
\text { uso de las } \\
\text { tecnologías de } \\
\text { información }\end{array}$} & $1^{\circ}$ & 0 & 0 & $45 \%$ & $40 \%$ & $15 \%$ \\
\hline & & $10^{\circ}$ & 0 & 0 & $35 \%$ & $50 \%$ & $15 \%$ \\
\hline \multirow{2}{*}{7} & \multirow{2}{*}{$\begin{array}{l}\text { Se me facilita la } \\
\text { información } \\
\text { apropiada }\end{array}$} & $1^{\circ}$ & $5 \%$ & $35 \%$ & $55 \%$ & $0 \%$ & $5 \%$ \\
\hline & & $10^{\circ}$ & $15 \%$ & $30 \%$ & $40 \%$ & $10 \%$ & $5 \%$ \\
\hline & $\begin{array}{l}\text { Promedio } 1^{\circ} \\
\text { semestre }\end{array}$ & & 0.1 & 1.7 & 6.6 & 8.7 & 2.9 \\
\hline
\end{tabular}

Fuente: Autor, Proceso de investigación

De acuerdo con los resultados expuestos en la tabla 1 se observa que en el ítem "evalúo la información que es apropiada para la toma de decisiones", ambos coinciden en la frecuencia "de acuerdo/casi siempre" con un $45 \%$. En el resto de las preguntas, las apreciaciones y opiniones entre los dos grupos fueron muy cercanas. Quizás donde hay un mayor distanciamiento es en el ítem de "clasifico la información disponible", donde los de primer semestre obtuvieron un $65 \%$ frente a un $40 \%$ del último, notándose además un decrecimiento para este ítem.

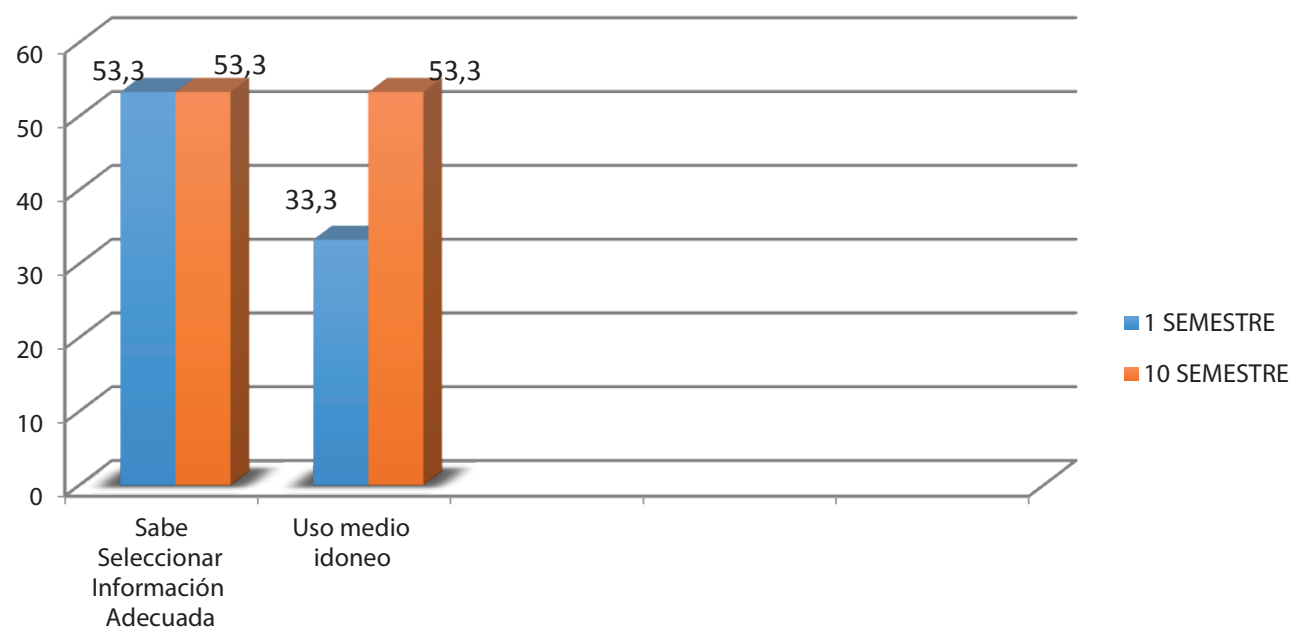

Figura 2

Cuestionario opinión y percepción Fuente: Autor, Proceso de investigación 
La figura 2 muestra los resultados en términos de promedios considerando los valores de las respuestas, lo que traducido en términos porcentuales indica que de los 20 estudiantes de primer semestre el $58 \%$ se encuentra en la frecuencia entre "de acuerdo/ casi siempre" y "totalmente de acuerdo/siempre". Esto permite inferir que según la apreciación de los mismos estudiantes esta competencia ha sido medianamente apropiada y desarrollada. Por otra parte, existe un importante porcentaje, equivalente al 33\%, que no presentan una respuesta clara, al optar por la opción "Indiferente/algunas veces".

Por otra parte, analizando las respuestas de los estudiantes del décimo semestre, los valores obtenidos no distan considerablemente de los estudiantes de primer semestre, incluso hay una disminución en el resultado, dado que según ellos también tienen cierto grado positivo de apropiación de esta competencia, y lo ratifica el $53 \%$ en la frecuencia obtenida, que se reparte entre las opciones "de acuerdo/casi siempre "y "totalmente de acuerdo/ siempre".

\section{Análisis de preguntas por quintiles}

En este apartado se analiza la categoría deductiva "selección y uso de la información" a través de las afirmaciones producto de los aspectos inductivos y que se aplicó a los estudiantes en el cuestionario diseñado utilizando la escala Likert. Esta información se analizó a partir de la agrupación de datos de la escala sobre la apropiación de la competencia genérica objeto de estudio en quintiles.

Se han dividido los quintiles en tres grupos: el primero $\left(1^{\circ}\right.$ y $\left.2^{\circ} \mathrm{Q}\right)$ comprende las categorías "casi nunca" y "nunca", el segundo $\left(3^{\circ} \mathrm{Q}\right)$ comprende la categoría "algunas veces" y el tercero $\left(4^{\circ}\right.$ y $5^{\circ}$ Q) comprende las categorías "casi siempre" y "siempre" (ver tabla 2).

El primer quintil corresponde a una visión negativa, el segundo quintil representa una posición de variabilidad de criterio o posición intermedia, y el tercer quintil refleja la visión positiva de dicha apropiación.

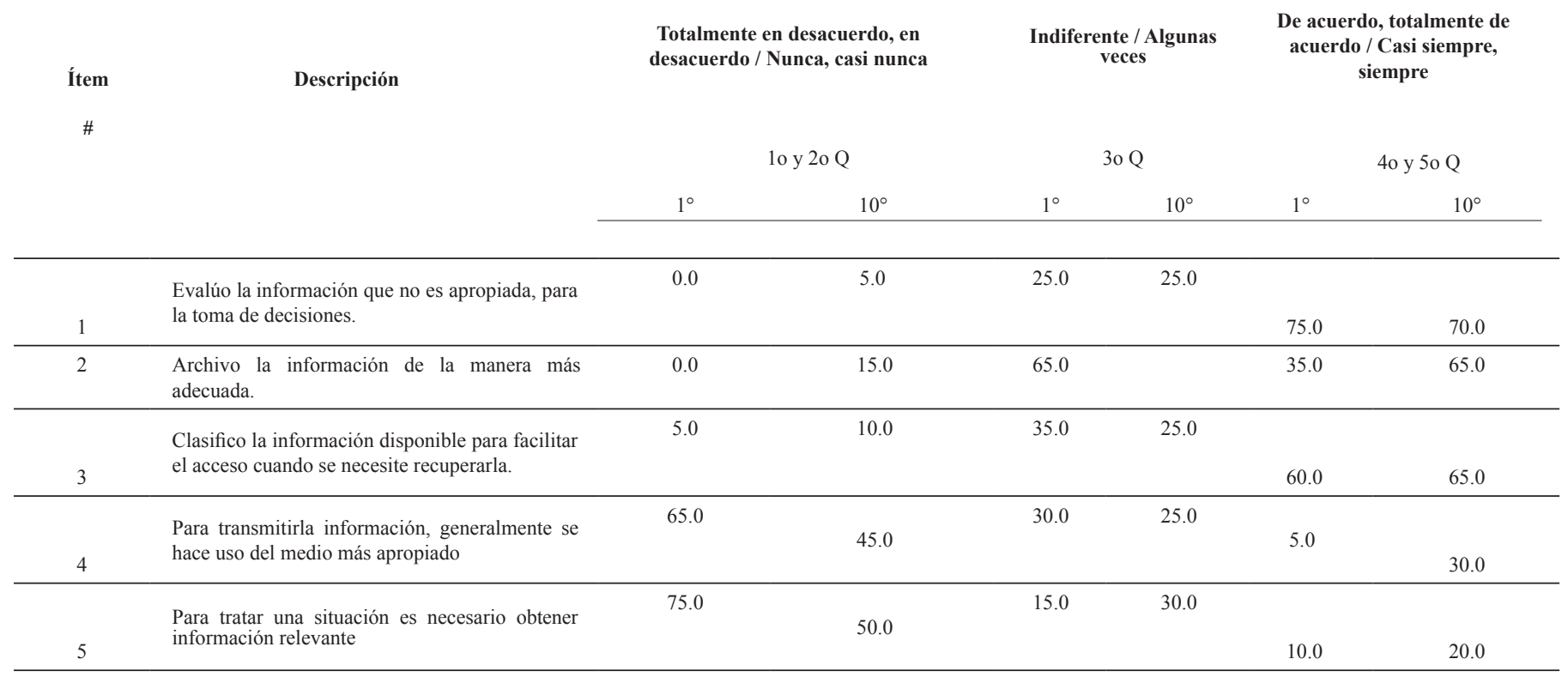




\begin{tabular}{|c|c|c|c|c|c|c|c|}
\hline 6 & $\begin{array}{l}\text { Poseo habilidades en el uso de las tecnologías de } \\
\text { la información. }\end{array}$ & 75.0 & 60.0 & 20.0 & 30.0 & 5.0 & 10.0 \\
\hline 7 & $\begin{array}{l}\text { Se me facilita seleccionar la información } \\
\text { apropiada. }\end{array}$ & 55.0 & 50.0 & 44.0 & 45.0 & 5.0 & 5.0 \\
\hline & TOTAL & 9.29 & 15.71 & 32.86 & 30.71 & 57.86 & 53.57 \\
\hline
\end{tabular}

Fuente: Autor, Proceso de investigación

De los totales de la tabla anterior se obtiene la figura 3, que facilita la comprensión de los resultados.

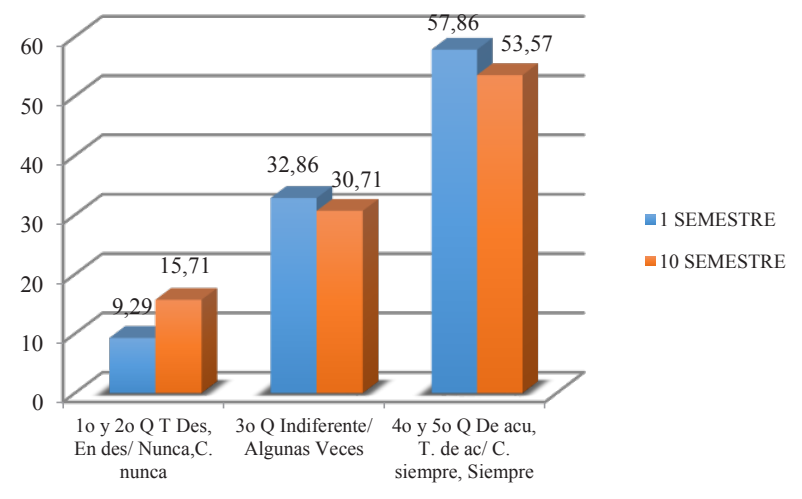

Figura 3

Quintiles selección y uso de la información

Fuente: Autor, Proceso de investigación

El resultado en el 4o y 5oQ, es superior entre los estudiantes de primer semestre que entre los de décimo; lo mismo ocurre en intermedia 3oQ, en el que se muestran mucho más vacilantes. Cabe anotar que aunque son valores a tener en cuenta, no modifican la percepción de la mayoría de los estudiantes de primero y décimo semestre, entre quienes existe una visión positiva en relación con la apropiación y desarrollo de la competencia.

La percepción de los estudiantes de primer semestre, por su parte, se encuentra ubicada en el 4o y 5oQ, con una visión positiva en relación con las tres categorías inductivas siguientes: evaluar la información que no es apropiada para la toma de decisiones, tratar una situación obteniendo la información relevante y poseer habilidades en el uso de las tecnologías de la información.

Asimismo, se destaca la vacilación que muestran los estudiantes de primer semestre en cuanto a la capacidad para archivar la información de la manera más adecuada. Esta categoría tiene un alto porcentaje en el $30 Q$, lo cual refleja con una tendencia intermedia de vacilación o incertidumbre.

De conformidad con los datos recopilados, es posible resaltar aquellas categorías inductivas que privilegian en su percepción los estudiantes de décimo semestre en cuanto a la apropiación y desarrollo de la información: Esta son: evaluar la información que es apropiada para la toma de decisiones, clasificar la información disponible para facilitar el acceso cuando se necesite recuperarla, tratar una situación obteniendo información relevante y poseer habilidades en el uso de las tecnologías de la información.

Finalmente, se observa una mayor seguridad de los estudiantes de décimo semestre en comparación con los de primer semestre, aunque no con la contundencia de criterio que deberían poseer, por haber tenido una mayor influencia del programa académico desarrollado a lo largo de su formación profesional.

\section{Análisis de las observaciones a partir de la pintura enriquecida}

Los estudiantes de ambos grupos se mostraron participativos e interesados durante esta actividad. Hubo buena interacción entre los participantes, aunque no por parte de todos en la misma medida.

Con es de esperar, los estudiantes de último semestre obtuvieron mejores resultados, dada su experiencia, su concepción del ejercicio mismo, y 
resultaron más proactivos, creativos y habilidosos en la selección de la información que debían compartir con sus compañeros y los investigadores, así como su pertinencia para la selección del medio idóneo para transmitir dicha información, palabras concretas, técnicas y apropiadas.

Entre los estudiantes de primer semestre se evidenció mayor timidez, miedo a arriesgarse, falta de organización, poca iniciativa, que afectaron sobre todo el uso del medio idóneo para transmitir la información del modo que el ejercicio lo exigía.

Frente a este instrumento, se diseñó una tabla que permitiera captar información en el momento en que los estudiantes desarrollaban esta actividad de grupo. Luego se tabularon los datos, los resultados se resumen en la figura 4.

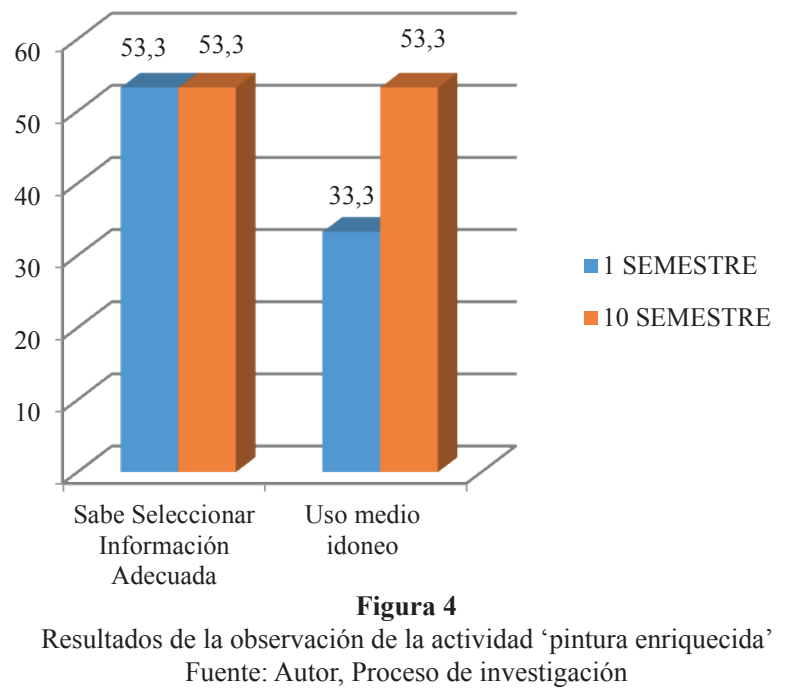

De la selección y uso de la información, los dos aspectos verificables elegidos por los investigadores fueron "sabe seleccionar información adecuada" y "uso del medio idóneo para transmitir la información".

Observando el desarrollo del ejercicio se puede decir que entre los alumnos del primer semestre se evidencia en un gran porcentaje de los sujetos, al igual que para el último semestre. Ambos grupos, en efecto, coinciden en un $53.3 \%$.

En cuanto al uso del medio idóneo para transmitir la información, en el primer semestre se observó que dicha competencia fue desarrolla durante el ejercicio planteado, tan solo por un $33.3 \%$, frente a un $53.3 \%$ del último semestre

Haciendo una comparación de los resultados se puede inferir que en la competencia "saber seleccionar información adecuada" no se evidencia un incremento del primer semestre frente del último semestre, lo que puede significar un ineficiente desarrollo de dicha competencia en el transcurso de la formación académica en la educación secundaria.

Con relación a la competencia "uso del medio idóneo" los resultados evidencian un crecimiento del 20\% del primer semestre frente al último, lo que significa que hay una evolución controlada en esta categoría.

\section{Entrevista a docentes}

Cuando se indagó entre los docentes sobre este aspecto, manifestaron que algunos estudiantes de primer semestre no reconocen información relevante de otra que no lo es. Así, la gran mayoría considera que hay muy pocos estudiantes que traen apropiada esta competencia al ingresar a la universidad, lo que significa que no son buenos clasificando, ni manejando información relevante. Esto se evidencia en los trabajos, en los talleres y en las mismas evaluaciones. Estos datos son corroborados por los resultados de $66.7 \%$ en el rango deficiente y tan solo un $33.3 \%$ en aceptable. 


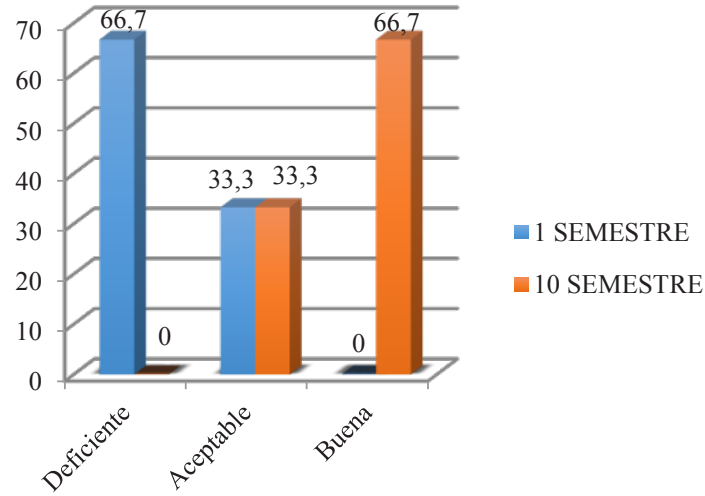

Figura 5

Resultados de la observación de la actividad 'pintura enriquecida' Fuente: Autor, Proceso de investigación

Por su parte, los estudiantes de décimo semestre consideran que, debido a la familiaridad que tienen con las tecnologías de la información, su paso por la universidad y la madurez obtenida con el transcurso de los años, están mucho más preparados para utilizar, clasificar, manipular y transmitir información, sin embargo, hay una tendencia a encasillar esta habilidad mayormente al uso de las TIC. Esta información la corrobora el $66.7 \%$ en el rango bueno y el 33\% en rango aceptable. 4 .

\section{Conclusiones}

La selección y uso de la información como competencia genérica ha tomado gran relevancia en los últimos años dado el vertiginoso avance de las tecnologías de la información y su incorporación en los ámbitos laborales, educativos y sociales.

Los resultados obtenidos mediante distintos instrumentos muestran esta competencia es considerada como fundamental para todos los estudiantes, independientemente del semestre que cursen. Sion embargo, el nivel de desarrollo de las habilidades asociadas a ella no son los más altos. En el cuestionario aplicado, por ejemplo, los estudiantes revelan una percepción medianamente positiva en la apropiación y desarrollo de esta competencia.
En la observación, desarrollada mediante la técnica de la pintura enriquecida, se privilegiaron dos aspectos: saber seleccionar información apropiada y el uso del medio idóneo para su transmisión. Los resultados señalan que hay un desarrollo significativo, sobre todo en lo que respecta al primer aspecto, en ambos grupos, pues tanto los estudiantes de primero como los del último semestre obtuvieron un porcentaje significativo. En cuanto al uso del medio idóneo, sí se evidenció una diferencia entre ambos grupos, sobresaliendo los de último. Ello permite inferir que hay un aprendizaje a lo largo de su paso por el programa de Ingeniería de Sistemas que se refleja en los resultados y en la actividad misma.

En la entrevista dirigida a los docentes, los resultados no favorecen a los estudiantes de primer semestre. En efecto, su apreciación frente a la posesión y desarrollo de esta competencia entre los alumnos de nuevo ingreso es que es deficitaria. No la han desarrollado suficientemente en su educación anterior, y ello se evidencia en el aula de clases, en los talleres y trabajos. En contraposición, los docentes aducen que los estudiantes de último semestre poseen capacidad suficiente para seleccionar y clasificar información, son creativos en el uso y selección del medio para transmitirla y poseen habilidades de las que se han podido apropiar durante su formación en el programa. Aun así, reconocen que todavía hay aspectos por mejorar. Estos resultados son coherentes con los reflejados en los diferentes estudios consultados y referidos en la presente investigación.

Finalmente, se espera que el presente estudio sirva como reflexión al interior de la Facultad de Ingeniería de la UPFS como punto de partida para el diseño de una propuesta curricular más acorde con el contexto nacional e internacional que contemple de una manera más nítida un modelo de educación superior por competencias. 


\section{Referencias}

Blanco, A. (2009). Desarrollo y evaluación de competencias en educación superior. Madrid: Narcea.

Comisión Europea - Programa Sócrates y Tempus. (2006). Una introducción a TuningEducational estructures in Europe: la contribución de las universidades al proceso de Bolonia. Bilbao: Ediciones de la Universidad de Deusto.

Comisión Europea - Programa ALFA. (2007). Reflexiones y perspectivas de la Educación Superior en América Latina. Informe final Proyecto Tuning América Latina 2004-2007. Bilbao: Ediciones de la Universidad de Deusto.

Corominas, E. (2001). Competencias genéricas en la formación universitaria. Revista de Educación, 9(12): 299-321.

Delors, J. (1995). Libro blanco sobre el crecimiento, competitividad y empleo. Retos y pistas para entrar en el siglo XXI. Bruselas: ANECA.

González, J., Wagenaar, R. yBeneitone, P. (2004). Tuning-América Latina: Un proyecto de las universidades. Revista Iberoamericana de Educación, 11(12), 151-164.

Ministerio de Educación Nacional. (2002). Las competencias en la educación superior. Disponible en: http://www.mineducacion. gov.co/1759/articles-189357_archivo_pdf_ introduccion.pdf .

Tejada, J. (1999). Acerca de las competencias profesionales.Herramientas, 56: 20-30. Disponible en http://academicos.iems.edu. $\mathrm{mx} /$ cired/docs/tg/macroacademiaquimica/ Competencias $\% 20$ profesionales TejadaFernandez.pdf

Tobón, S. (2005). Formación basada en competencias: pensamiento complejo, diseño curricular y didáctica. Bogotá: ECOE.

Universidad Francisco de Paula Santander Ocaña UFPSO. (s.f.). Oferta Académica UFPS Ocaña. Obtenido de Ingeniería de Sistemas. Misión: https://ufpso.edu.co/ofertaufpso/sistemas/ Mision 